\title{
Shielding membrane surface carboxyl groups by covalent-binding graphene oxide to improve anti-fouling property and the simultaneous promotion of flux
}

\author{
Jing-Long Han ${ }^{\text {a, b }}$, Xue Xia ${ }^{\text {a, }}{ }^{* *}$, Yu Tao ${ }^{c}$, Hui Yun ${ }^{\text {a, b }}$, Ya-Nan Hou ${ }^{\text {d }}$, Chang-Wei Zhao ${ }^{\text {, }}$ \\ Qin Luo a , Hao-Yi Cheng a , Ai-Jie Wang a, d, * \\ ${ }^{a}$ Research Center for Eco-Environmental Sciences, Chinese Academy of Sciences, Beijing 100085, PR China \\ ${ }^{\mathrm{b}}$ University of Chinese Academy of Sciences, Beijing 100049, PR China \\ ${ }^{\mathrm{c}}$ Department of Chemical Engineering, Imperial College London, South Kensington Campus, London SW7 2AZ, UK \\ d State Key Laboratory of Urban Water Resource and Environment, Harbin Institute of Technology, Harbin 150090, PR China
}

\section{A R T I C L E I N F O}

\section{Article history:}

Received 13 March 2016

Received in revised form 18 May 2016

Accepted 13 June 2016

Available online 16 June 2016

\section{Keywords:}

Membrane modification

Combined membrane fouling

Membrane filtration

Wastewater secondary effluent treatment

Volume charge density

Zeta potential

\begin{abstract}
A B S T R A C T
Graphene oxide (GO) is an excellent material for membrane surface modification. However, little is known about how and to what extent surface functional groups change after GO modification influence membrane anti-fouling properties. Carboxyl is an inherent functional group on polyamide or other similar membranes. Multivalent cations in wastewater secondary effluent can bridge with carboxyls on membrane surfaces and organic foulants, resulting in serious membrane fouling. In this study, carboxyls of a polydopamine (pDA)/1,3,5-benzenetricarbonyl trichloride (TMC) active layer are shielded by covalently-bound GO. The process is mediated by $\mathrm{N}$-(3-dimethylaminopropyl)-N'-ethylcarbodiimide (EDC)/N-hydroxysuccinimide (NHS). For GO containing low quantities of carboxyls, X-ray photoelectron spectroscopy (XPS) and zeta potential analyzer test results reveal that the carboxyl density decreased by $52.3 \%$ compare to the pDA/TMC membrane after GO modification. Fouling experiments shows that the flux only slightly declines in the GO functionalized membrane (19.0\%), compared with the pDA/TMC membrane (36.0\%) after fouling. In addition, during GO modification process the pDA/TMC active layer also become harder and thinner with the aid of EDC/NHS. So the pure water permeability increases from $56.3 \pm 18.2$ to $103.7 \pm 12.0 \mathrm{LMH} / \mathrm{MPa}$. Our results provide new insights for membrane modification work in water treatment and other related fields.
\end{abstract}

(c) 2016 Elsevier Ltd. All rights reserved.

\section{Introduction}

Membrane fouling limits the more widespread applications of nanofiltration (NF) or precise ultrafiltration (UF) membranes in water or wastewater treatment (Zhao and Yu, 2014). The membrane fouling caused by organic foulants in wastewater secondary effluent and some natural water is a difficult problem (Hong and Elimelech, 1997). For membranes surface with much carboxyls, the fouling process can be greatly enhanced by low concentrations of $\mathrm{Ca}^{2+}$ or other multivalent cations, because these cations can effectively coordinate with the carboxyls on the membrane surface

\footnotetext{
* Corresponding author. CAS Key Lab for Environmental Biotechnology, Research Center for Eco-Environmental Sciences, PR China.

** Corresponding author.

E-mail addresses: xuexia@rcees.ac.cn (X. Xia), ajwang@rcees.ac.cn (A.-J. Wang).
}

and organic foulants (Mo et al. 2011, 2012). As $\mathrm{Ca}^{2+}$ or other multivalent cations widely coexist with organic foulants in actual wastewater (Lu et al., 2013), reducing the carboxyls density on membrane surface can be a feasible choice to control this type of membrane fouling.

Carboxyl is an inherent group on polyamide (PA) membranes or other similar membranes, which can originate from the hydrolysis of unreacted acyl chloride groups during active layer preparation (Mo et al., 2012). Carboxyls can be the source of fixed charges in the membrane active layer after partial hydrolysis (Childress and Elimelech, 2000, 1996), and fixed charges have an important influence on the rejection efficiency of nanofiltration (NF) or similar precise ultrafiltration (UF) membranes (Szymczyk and Fievet, 2005). Above all, during the active layer formation, the excess numbers of acyl chloride groups may be favorable to the formation of three-dimensional active layer structures. The experiment 
described in previous work revealed the density of carboxyl groups could be decreased by selecting monomers with fewer acyl chloride groups to form the polyamide layer (Mo et al., 2012). But this method may also have an influence on the membrane divalent ion separation efficiency and flux (Mo et al., 2012). In addition, surface carboxyl groups can also be used for surface modification (Jie et al., 2015; Li et al., 2016; Yu et al., 2016).

Membrane active layer modification can improve membrane anti-fouling properties. The existing modification methods mainly include surface coating, surface grafting, incorporation of hydrophilic monomers/inorganic particles and zwitterionic modification (Zhao and Yu, 2014). However, in some cases, membrane flux, rejection efficiency, robustness and anti-fouling properties are mutually exclusive. Therefore, membrane modification methods may influence the water permeability (Lu et al., 2013; Van Wagner et al., 2011; Liu et al., 2015), surface roughness (Van Wagner et al., 2011), or rejection efficiency (An et al., 2011; Ye et al., 2015). Materials possessing better characteristics may help to handle these problems better.

Graphene oxide (GO) is an excellent material for membrane modification (Hegab and Zou, 2015). Water can nearly unimpeded permeated though graphene two-dimensional nanocapillaries (Nair et al., 2012). GO has other unique qualities, including ultrahigh strength (Lee et al., 2008), atomic-scale thickness (Novoselov et al., 2004), high antibacterial property and low cytotoxicity (Hu et al., 2010; Tu et al., 2013), and the possibility of producing ultraflat surface (Lui et al., 2009). It also possesses good chemical stability (Dreyer et al., 2010) and hydrophilic properties (Nair et al., 2012; Dreyer et al., 2010), and can be prepared from inexpensive graphite (Hu et al., 2010; Hummers and Offeman, 1958). Furthermore, there are abundant oxygen-containing functional groups on GO available for membrane modification. Several membrane surface modification reports have been published that use GO to improve flux (Chae et al., 2015; Berean et al., 2015), increase antibiofouling properties (Hegab et al., 2015; Perreault et al., 2014; Zou et al., 2016) and enhance chlorine resistance properties (Choi et al., 2013). Previous studies also found that after surface modification by GO, the membrane anti-fouling properties to the protein, alginate or humic acid foulants were obviously improved (Hegab et al., 2015; Choi et al., 2013; Hu et al., 2016; Han et al., 2015). And the improvement of fouling resistance was attributed to hydrophilicity increase and surface roughness reducing (Hegab et al., 2015; Choi et al., 2013; Hu et al., 2016; Han et al., 2015). Even though GO have demonstrated effective adsorption performance (Perreault et al., 2015), it was explained that the organic foulants were adsorbed mainly on the basal plane of GO nanosheets while water enters in the GO modified membrane surface primarily around the oxidized edges of GO nanosheets (Hu et al., 2016). However, to the best of the authors' knowledge, there is little report on whether membrane surface functional groups change after GO surface modification influence membrane anti-fouling properties or not. A better understanding of these processes will help to the development of GO surface modification applications. Carboxyl group are present in low quantities at the periphery of moderately oxidized GO (Nair et al., 2012; Dreyer et al., 2010; Perreault et al., 2014). Here, we hypothesize that by covering carboxyls on the membrane active layer surface with GO nanosheets, $\mathrm{Ca}^{2+}$ bridged fouling could be significantly mitigated, whereas the rejection efficiency would not be affected because the volume charge density is maintained by protecting the carboxyls in the active layer using GO.

Novel polydopamine (pDA)/1,3,5-benzenetricarbonyl trichloride (TMC) composite membranes have the enormous potential for application in wastewater treatment and other fields (Zhao et al., 2014) because of their excellent structural and chemical stability (especially their chlorine resistance properties (Zhao et al.,
2014)). Dopamine can be easily self-deposited on nearly all types of inorganic and organic substrates with controllable thickness (Hu and Mi, 2013), and pDA has excellent durability (Liu et al., 2014). The amino and hydroxyl groups on the polydopamine can react with acyl chloride groups of TMC and other similar compounds to form amide bonds and ester bonds, constituting the active layer (Zhao et al., 2014; Hu and Mi, 2013; Liu et al., 2014). Thus, the pDA/ TMC composite membrane is a good candidate membrane to investigate the relationship of how the surface carboxyl groups change affect the anti-fouling properties after surface modification by GO. As the pDA/TMC active layer could be low in roughness, high in hydrophilic properties (Zhao et al., 2014) the influence of these two aspects can be excluded easier. In addition, the controllable auto-polymerized membrane avoids uncontrolled errors during manually scraping membrane (Mo et al., 2012).

$N$-(3-Dimethylaminopropyl)- $N^{\prime}$-ethylcarbodiimide $\quad$ (EDC)/Nhydroxysuccinimide (NHS) can be used to convert the native carboxyl groups of the polyamide surface into intermediate aminereactive esters to covalently-bind GO or carbon nanotubes to membrane surface (Perreault et al., 2014; Tiraferri et al., 2011). EDC/NHS is also widely used to catalyze amide-crosslinks between carboxyl groups and amino groups (Wissink et al., 2001; Peng et al., 2013; Staros et al., 1986). The decline of membrane flux during the early stage of filtration is significantly affected by membrane compaction (Petersen, 1993). Thin-film composite (TFC) membrane compaction can occur in both the supporting layer and active layer. As the flux of the TFC membranes is controlled by the active layer (Petersen, 1993), Hussain et al. stated that the surface pore collapse may be the key mechanism for membrane compaction (Hussain et al., 2013). If EDC/ NHS can enhance the amide-crosslinks between carboxyl groups and amino groups of pDA/TMC active layer, and suppress the surface pore collapse, the membrane flux can be promoted.

In this study, we use an environmentally friendly method to covalently-bind GO to the surface of pDA/TMC active layer, by EDC/ NHS mediated interfacial polymerization to shield, but not remove, the carboxyls in the membrane active layer. We aim to simultaneously promote the anti-fouling property and flux, and maintain the membrane rejection efficiency.

\section{Material and methods}

\subsection{GO preparation}

GO was prepared from flaky graphite using the Hummers' method (Hummers and Offeman, 1958; Marcano et al., 2010). The high-temperature oxidation process at $98{ }^{\circ} \mathrm{C}$ was shortened to obtain moderately oxidized graphite oxide. The graphite oxide was washed using ultrapure water (Milli-Q) until the $\mathrm{pH}$ of the supernatant stabilized. Ultrasonic exfoliation ( $40 \mathrm{kHz}, 100 \mathrm{~W}$, Kun Shan Ultrasonic Instruments, China) of the GO nanosheets was performed in an ice bath for $1 \mathrm{~h}$. The prepared GO solution was stored at $4{ }^{\circ} \mathrm{C}$. Prior to use, the stock solution of GO was indirectly ultrasonicated for $10 \mathrm{~min}$ and diluted to the required concentration. The details of GO characterization methods and results (Figs. S1-S7) are listed in the Supplementary Information section.

\subsection{Brief description of membrane fabrication}

A new method was developed to prepare the GO functionalized pDA/TMC TFC membranes (Fig. 1). The main reactions and detailed description of the TFC membrane fabrication are provided in the Supplementary Information section. Briefly, a poly(vinylidene fluoride) (PVDF) microfiltration membrane (Shanghai SINAP Membrane Tech, Shanghai, China) with an average surface pore diameter of $80 \mathrm{~nm}$ was used as supporting membrane. The pDA/ 
TMC TFC membrane was fabricated by forming a pDA/TMC active layer on the surface of the PVDF membrane (known as pDA/TMC), followed by covalent-binding of $\mathrm{GO}$ on the active layer to obtain the GO functionalized pDA/TMC membrane (known as pDA/TMC/GO or PVDF/pDA/TMC/GO), using a method developed from previous reports (Perreault et al., 2014; Tiraferri et al., 2011). The layer-by-layer method was introduced to increase the amount of GO bound to the membrane surface (Sanyal and Lee, 2014). The rotation infusion Ethylenediamine (ED) and activated GO solutions were repeated 10 and 15 times to obtain multi-layer GO modified pDA/TMC membranes (known as pDA/TMC/G010 or PVDF/pDA/TMC/G010, pDA/ TMC/G015 or PVDF/pDA/TMC/GO15).

To confirm the effectiveness of the GO modification method and obtain a clearer scanning electron microscope (SEM) image, a polyethersulfone (PES) microfiltration membrane (HangZhou DUTE Filter Company, China) was also used as the supporting membrane, with the same method described above (known as PES/pDA/TMC/ GO10) to prepare the GO modification membrane. The PES microfiltration membrane possesses a larger pore size (in general $0.5-2 \mu \mathrm{m}$ ) than the PVDF supporting membrane, so the pDA/TMC active layer cannot coated on the micropores of PES supporting membrane well, and GO nanosheets were easier to be distinguished using SEM.
pDA/TMC/C10 membranes were measured at a resolution of $4 \mathrm{~cm}^{-1}$ and excitation wavelength of $532 \mathrm{~nm}$. Au colloid (Sun et al., 2014) was dropwise added to the membrane samples and dried at $45{ }^{\circ} \mathrm{C}$ to make up the surface-enhanced Raman scattering (SERS) to enhance the spectra signals.

The surface functional groups of the dried membranes were determined using X-ray photoelectron spectroscopy (XPS, Thermo Scientific Escalab 250Xi, UK) using $300 \mathrm{~W}$ of $\mathrm{Al} \mathrm{K} \alpha$ radiation at approximately $3 \times 10^{-9}$ mbar. The bond energies were referenced to the $\mathrm{C} 1 \mathrm{~s}$ line at $284.8 \mathrm{eV}$ from adventitious carbon and the $-\underline{\mathrm{C}}-\mathrm{F}$ line at $290.35 \mathrm{eV}$ from the PVDF supporting membrane. After the $\mathrm{C} 1 \mathrm{~s}$ peak of the membrane was deconvoluted, the area percentages of the functional groups in the active skin layer were calculated. To obtain a more accurate result, the carbon atoms in the supporting layer were removed. The chemical formula of PVDF supporting layer is $-\left(\mathrm{CH}_{2}-\mathrm{CF}_{2}\right)_{n}-$. So when there was a unit of $-\mathrm{C}-\mathrm{F}$ peak area showed in XPS $\mathrm{C} 1 \mathrm{~s}$ peak, there was a unit of $-\mathrm{C}-\mathrm{C}-$ peak area belonged to PVDF supporting layer also showed accompanying. Therefore, there was twice as many carbon atoms in the PVDF supporting layer should be subtracted compared with the $-\mathrm{C}-\mathrm{F}$ area percentages. For example, the carboxyl area percentage in the pDA/TMC active skin layer was calculated as

$$
\text { Area percentage }(\mathrm{COOH})=\frac{\text { Area }(\mathrm{COOH})}{\operatorname{Area}((\mathrm{C}-\mathrm{C} / \mathrm{C}=\mathrm{C})+(\mathrm{C}-\mathrm{N})+(\mathrm{C}=\mathrm{O})+(\mathrm{C}-\mathrm{O}-\mathrm{C} / \mathrm{C}-\mathrm{OH})+(\mathrm{COOH})+(\mathrm{C}-\mathrm{F}))-2 \text { Area }(\mathrm{C}-\mathrm{F})} \times 100 \%
$$

To elucidate the impact of the GO modification and EDC/NHS catalyst on the anti-fouling and filtration properties of the membrane, control membranes without a GO layer (known as pDA/TMC) $\mathrm{C} 10$ or $\mathrm{PVDF} / \mathrm{pDA} / \mathrm{TMC} / \mathrm{C} 10)$ were fabricated following the same procedure mentioned above, with the exception of replacing the GO solution with ultrapure water.

\subsection{Membrane characterization}

The microstructure of membrane surfaces was observed using a field emission scanning electron microscope (FESEM, Hitachi SU8020, Japan). The samples were dried in an oven at $45{ }^{\circ} \mathrm{C}$ and sprayed with $\mathrm{Au}$ for $10 \mathrm{~s}$ under fine vacuum conditions, prior to observation. The Raman spectra (LabRam HR Evolution of Hoeiba Jobin Yvon, France) of the pDA/TMC/GO10, pDA/TMC/GO15 and
The zeta potential of the membrane surfaces was determined using a SurPASS electrokinetic analyzer with an adjustable gap cell (cell height of $100 \pm 10 \mu \mathrm{m}$, Anton Paar GmbH, Austria) (Buksek et al., 2010). Measurements were carried out in a $1 \mathrm{mM} \mathrm{KCl}$ solution at $25 \pm 1{ }^{\circ} \mathrm{C}$. The $\mathrm{pH}$ was set in the range of $4-10$ by adding $\mathrm{HCl}$ or $\mathrm{KOH}$. Prior to the measurement, the samples were equilibrated with the testing solution for at least $45 \mathrm{~min}$ (Ben-Sasson et al., 2014). The original Helmholtz-Smoluchowski approach was applied to calculate the zeta potential (Buksek et al., 2010).

To get the cross section view of membrane active layer, the cross-sectional transmission electron microscope (TEM) images of different membranes were taken using ultramicrotomy. The samples for ultramicrotomy were firstly embedded in Eponate resin and solidified for $48 \mathrm{~h}$ at $60{ }^{\circ} \mathrm{C}$. The embedded samples were sliced to pieces with the thickness of $\sim 70 \mathrm{~nm}$ by an ultramicrotome
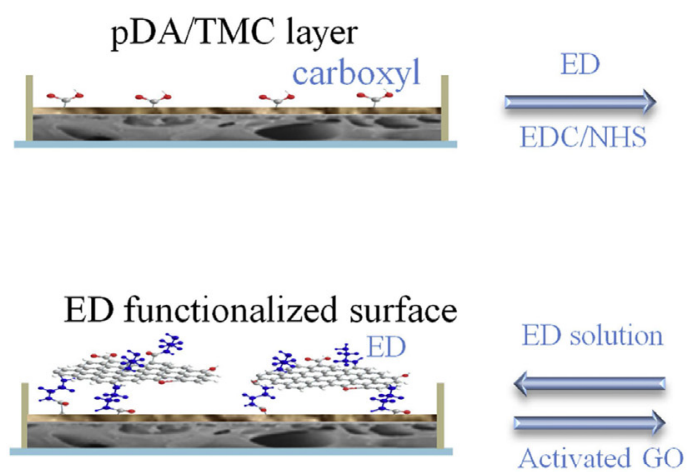
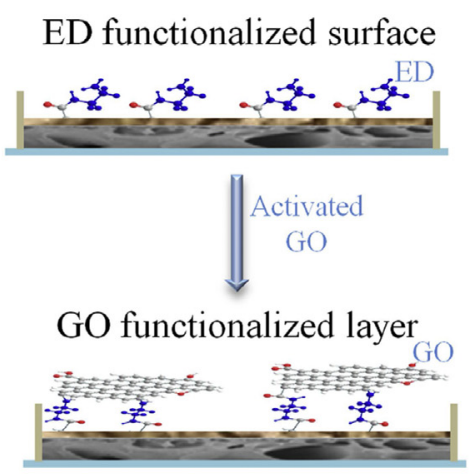

Fig. 1. The main procedure to prepare GO functionalized membranes. 
(Ultracut UCT, Leica Microsystems IR GmbH, Germany) and observed by an TEM (JEM-1400 from JEOL Ltd.) at $80 \mathrm{kV}$.

\subsection{Membrane compaction and rejection performance test}

The membrane compaction process, water permeability, rejection performance were tested on a modified SEPA CF II membrane cell system (Sterlitech Corporation, USA) with an effective membrane area of $137.1 \mathrm{~cm}^{2}$. All of these tests were conducted at a transmembrane pressure (TMP) of $0.45 \mathrm{MPa}$ and a cross-flow velocity of $0.132 \mathrm{~m} / \mathrm{s}$. The temperature of the feed solution was $26 \pm 1^{\circ} \mathrm{C}$. Ultrapure water was used for membrane compaction and water permeability measurement. The flux before a certain time was manually recorded to represent the compaction process. When flux change was stable, the water permeability, $A_{k}$, was then calculated as:

$\mathrm{A}_{\mathrm{k}}=\frac{\Delta \mathrm{V}}{\mathrm{A}_{\mathrm{m}} \Delta \mathrm{P} \Delta \mathrm{t}}$

Where, $A_{m}$ is the effective membrane surface area, $\Delta V$ is the collected permeate volume during $\Delta \mathrm{t}$, and $\Delta \mathrm{P}$ is transmembrane pressure (TMP).

Rejection performance was characterized by solutions of Alizarin red $\mathrm{S}(10 \mathrm{mg} / \mathrm{L})$, chromotropic acid disodium $(10 \mathrm{mg} / \mathrm{L})$ and Rhodamine $\mathrm{B}(10 \mathrm{mg} / \mathrm{L})$ with the $\mathrm{pH}$ adjusted to 7.0 . The concentrations of the Alizarin red S, chromotropic acid disodium salt and Rhodamine B were measured using a spectrophotometric method with a BeckMan Coulter DU730 spectrophotometer (Beckman Coulter, Inc, USA) at wavelengths of 261, 233 and $553 \mathrm{~nm}$, respectively. The apparent rejection to organic matter, $\mathrm{R}$, was calculated from:

$\mathrm{R}=100 \% \times\left(1-\frac{C_{p}}{C_{F}}\right)$

Where, $C_{p}$ and $C_{F}$ are the concentrations of tested organic matter in the permeated and feed solutions, respectively.

\subsection{Anti-fouling property test}

Membrane fouling testing was performed in the same modified SEPA CF II membrane cell system mentioned in Section 2.4 using a previously reported process (Mo et al., 2012; Choi et al., 2013; Han et al., 2015). The $\mathrm{Ca}^{2+} /$ organic foulants compound synthetic wastewater was constituted by $\mathrm{CaCl}_{2}, \mathrm{NaCl}, \mathrm{NaHCO}_{3}$ and sodium alginate (Mo et al., 2012; Lu et al., 2013). Before fouling, the compacted membranes were equilibrated with a background electrolyte containing $1 \mathrm{mM} \mathrm{CaCl}_{2}, 16 \mathrm{mM} \mathrm{NaCl}$ and $1 \mathrm{mM} \mathrm{NaHCO}_{3}(\mathrm{Mo}$ et al., 2012) until the flux stabilized. The cross-flow velocity during this stage was $0.11 \mathrm{~m} / \mathrm{s}$. The same TMP of $0.45 \mathrm{MPa}$ was chosen to exclude the impact of TMP on membrane fouling (Xie et al., 2015). Alginate was used as a model macromolecule for the fouling experiment. Sodium alginate solution was added to the background solution to achieve an alginate concentration of $20 \mathrm{mg} /$ $\mathrm{L}$ to initialize the fouling stage. The fouling experiment was performed until the filtrate was more than $600 \mathrm{ml}$. Physical cleaning was performed to evaluate fouling reversibility. The cross-flow velocity was increased to $0.44 \mathrm{~m} / \mathrm{s}$, and the hydraulic pressure was kept close to zero. The composition of the cleaning solution was same as the background electrolyte. After $30 \mathrm{~min}$ of physical cleaning, the same background electrolyte was used again to determine the flux of a cleaned membrane. The cleaning efficiency was calculated as previous reports (Mo et al., 2012).

\section{Results and discussion}

\subsection{Physicochemical properties of $G O$}

Single-layer GO nanosheets were successfully prepared at thicknesses ranging from 0.8 to $1.1 \mathrm{~nm}$ (Fig. S1), and the plane size of GO was approximately $1-6 \mu \mathrm{m}$ (Fig. S2). As GO nanosheets tend to be planar superimposed when concentration reach to a certain level, they can only be recognized by wrinkles in the FESEM image (Fig. S3). The Raman spectra exhibited characteristic D peaks at $\sim 1350 \mathrm{~cm}^{-1}$, which are associated with the order/disorder of the system, and $G$ peaks at $1590 \mathrm{~cm}^{-1}$, which are an indicator of the stacking structure (Dreyer et al., 2010; Das et al., 2008) (Fig. S4). The zeta potential measurement revealed that the GO nanosheets were negatively charged $(<-60 \mathrm{mV})$ and stably dispersed in the water over a wide $\mathrm{pH}$ range of $4-10$ (Fig. S5) (Riddick, 1968). This negative potential likely originated from the selective adsorption of $\mathrm{OH}^{-}$or $\mathrm{Cl}^{-}$on GO rather than the hydrolysis of the carboxyl, as the zeta potential did not become more negatively charged with the increase in pH (Fig. S5).

XPS spectra and Fourier transform infrared spectroscopy (FTIR) spectrum (Fig. S6) of GO (Fig. S7, Table S1) were also obtained. After deconvolution of the XPS data (Table S1), the obtained GO nanosheets were found to be abundant in $-\mathrm{C}-\mathrm{OH} /-\mathrm{C}-\mathrm{O}-\mathrm{C}$ (aromatic) groups (42.9\%). The $-\mathrm{COOH}$ area percentage in each side of the $\mathrm{GO}$ nanosheets was only $1.2 \%$. This revealed the tremendous potential of using GO to shield membrane surface carboxyls.

\subsection{Formation of $\mathrm{pDA} / \mathrm{TMC}$ active layer}

As revealed by FESEM, the surface of the pDA/TMC TFC membrane (Fig. 2b) was basically with no micropore, different from the pristine PVDF membrane (Fig. 2a), indicating that the pDA/TMC active layer formed after the dopamine and TMC treatment (Fig. $2 \mathrm{~g}-\mathrm{i}$ ). At the same time, the water permeability decreased sharply from $5487.8 \pm 398.5 \mathrm{~L} /\left(\mathrm{m}^{2} \cdot \mathrm{h} \cdot \mathrm{MPa}\right)$ to $56.3 \pm 18.2 \mathrm{~L} /$ $\left(\mathrm{m}^{2} \cdot \mathrm{h} \cdot \mathrm{MPa}\right)$ (Fig. 5a). The pDA/TMC active layer formation was further supported by the ATR-FTIR differential spectra (Fig. S12), which showed that an amide peak emerged at approximately $1720 \mathrm{~cm}^{-1}$ (Zhao et al., 2014), after reacting with TMC.

\subsection{Irreversible binding of GO}

The GO characteristic peaks ( $G$ peaks at $\sim 1590 \mathrm{~cm}^{-1}$ and D peaks at $\sim 1350 \mathrm{~cm}^{-1}$ ) were captured in the Raman spectra (Fig. 3) after GO functionalization. Moreover, the intensities of the $G$ and $D$ peaks increased by $50 \%$, coupling with the GO functionalization times increased from 10 to 15 . Furthermore, GO characteristic folds also emerged on the surface of the membrane after GO functionalization (Fig. 2c). Clearer GO images (Fig. 2e, f) were also obtained when the PES microfiltration membrane with larger pore sizes $(0.5-2 \mu \mathrm{m})$ was used as the supporting membrane to prepare GO functionalized membranes. The FESEM images and Raman spectra confirmed the effectiveness of the GO functionalization method.

\subsection{Membrane surface carboxyl shielding}

The functional group ratios of GO, pDA/TMC and pDA/TMC/C10 membrane active layers are listed in Table S1 and Fig. 4a-d after $\mathrm{C} 1 \mathrm{~s}$ peak was deconvoluted and calculated. As shown in Fig. $4 \mathrm{a}-\mathrm{d}$ and Table S1, the average - $\mathrm{COOH}$ density in the pDA/TMC/GO layer decreased from $4.4 \%$ to $2.1 \%$ compared with the pDA/TMC active layer in the pDA/TMC membrane. Furthermore, from the Table S1 and Fig. 4b-d, we can also find that during GO functionalization process, the covalent-binding GO on the membrane surface was 

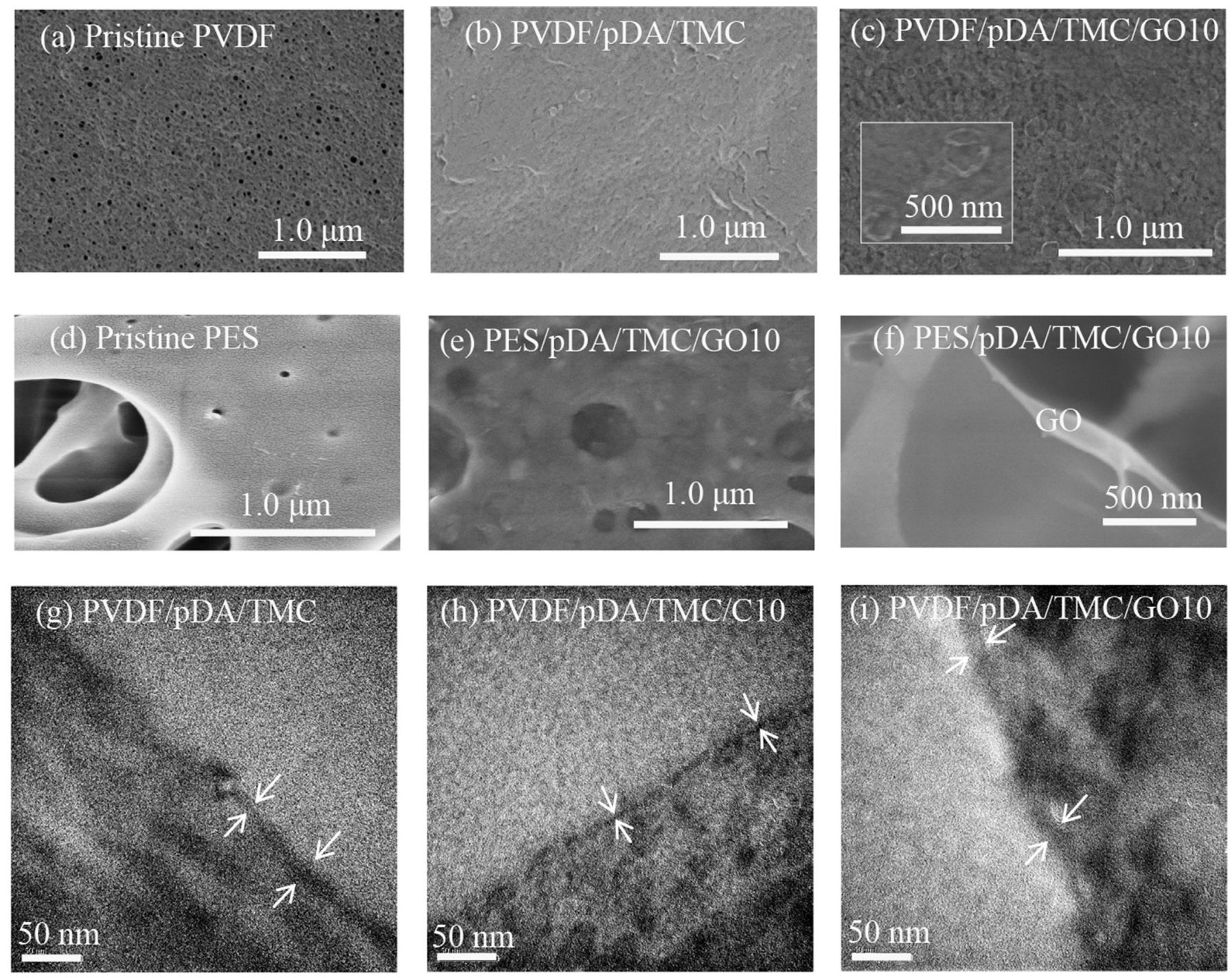

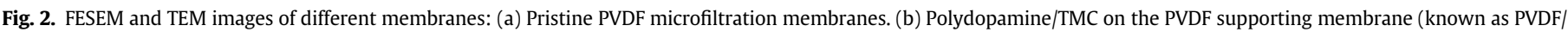

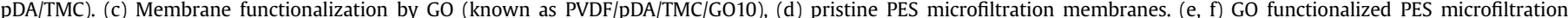

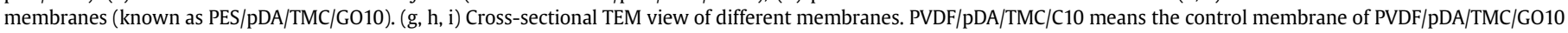
without GO addition.

mainly aided by the $-\mathrm{C}-\mathrm{N}-$ bonds that formed from the ED and epoxy of the GO (Hung et al., 2014) (see the discussion in Supplementary Information). However, a small amount of $-\mathrm{COOH}$ also formed to amide bond with the ED aided by EDC/NHS (Perreault et al., 2014). Therefore, the remaining $-\mathrm{COOH}$ on one side of the GO could be less than $1.2 \%$. In addition, GO nanosheets are a two-dimensional material, and the carboxyls tend to be homogeneously distributed on the two side of the nanosheets. As the XPS data only revealed the average value of $-\mathrm{COOH}$ density at a certain depth, not at the outer surface of the pDA/TMC/GO layer. Therefore, the reality - $\mathrm{COOH}$ density in the outer surface could be less than $2.1 \%$.

Zeta potential analyzer results also revealed membrane surface $-\mathrm{COOH}$ shielded by the GO (Fig. 4e). Compared with the relative stable zeta potential of the pDA membrane containing no $-\mathrm{COOH}$, all of the $-\mathrm{COOH}$-containing membranes (pDA/TMC, pDA/TMC/ $\mathrm{C} 10$ and $\mathrm{pDA} / \mathrm{TMC} / \mathrm{GO} 10$ ) become more negatively charged in their zeta potential along with the increase in $\mathrm{pH}$. This demonstrated that the dissociation of $-\mathrm{COOH}(\mathrm{pKa}=\sim 3.5$, predicted by $\mathrm{ACD} / \mathrm{Labs}$ ) on the membrane surface was the main contributor to the change in the surface zeta potential, so the zeta potential can be an indicator of the $-\mathrm{COOH}$ density on the membrane surface (Childress and Elimelech, 2000, 1996). The zeta potential of the pDA/TMC $(-54.1 \mathrm{mV})$ and the pDA/TMC/C10 $(-53.5 \mathrm{mV})$ membranes were both considerably more negatively charged than that of the pDA/ TMC/GO10 membrane $(-45.1 \mathrm{mV})$ at $\mathrm{pH} 7$, which provided further evidence that the $-\mathrm{COOH}$ on the membrane surface was effectively shielded by the GO during the membrane modification.

The GO nanosheets were expected to shield only the outer surface of the pDA/TMC active layer, meaning that the carboxyls under the GO layer could still contribute to the volume charge density of the active layer after partial hydrolysis (Childress and Elimelech, 2000, 1996). The volume charge density had an important effect on the rejection efficiency of nanofiltration (Szymczyk and Fievet, 2005) and was not easy to be tested. However, this effect can be indirectly discussed using the rejection efficiency results.

The membrane rejection efficiency to carefully select organics could reveal the volume charge density of the active layer. The rejection efficiencies of $\mathrm{pDA} / \mathrm{TMC} / \mathrm{GO} 10$ and $\mathrm{pDA} / \mathrm{TMC} / \mathrm{C} 10$ for 


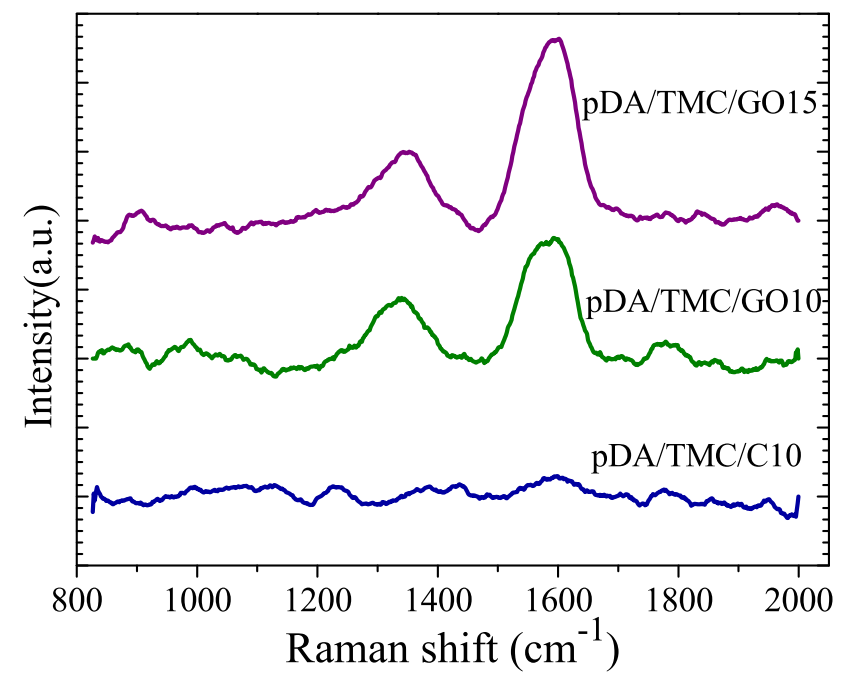

Fig. 3. Raman spectra recorded from the $\mathrm{pDA} / \mathrm{TMC} / \mathrm{GO}$ membranes and the control membrane.

chromotropic acid disodium (with two negative charges at $\mathrm{pH} 7$ ) were $90.5 \pm 2.5 \%$ and $87.3 \pm 4.2 \%$, respectively, and the rejection efficiencies were $81.6 \pm 0.4 \%$ and $81.8 \pm 0.8 \%$ for Alizarin Red S (with one negative charge at $\mathrm{pH} 7$ ), respectively. For Rodamine B (an apparent positive charge at $\mathrm{pH} 7$ ), the apparent rejection efficiencies of $\mathrm{pDA} / \mathrm{TMC} / \mathrm{GO} 10$ and $\mathrm{pDA} / \mathrm{TMC} / \mathrm{C} 10$ were $11.4 \pm 6.4 \%$ and $4.5 \pm 1.1 \%$, respectively. The ionic weights of chromotropic acid disodium, Alizarin Red S and Rodamine B were 318.28, 319.27 and 443.51, respectively. Therefore, the number of negative charges directly affected the level of apparent rejection efficiency, and the role of ionic weights was limited to the rejection for these three organic compounds. The apparent rejection efficiency also revealed that the effective pore size of the membrane active layers in this study was larger than Stokes' radius for the Rhodamine B ion. Furthermore, there was a high volume charge density in the active layers according to the Donnan-Steric partitioning pore model (Szymczyk and Fievet, 2005; Bowen and Mukhtar, 1996; Vezzani and Bandini, 2002). Therefore, the apparent rejection efficiency remains constant after the GO functionalization revealed that the volume charge density remains constant. This means that after carboxyls in the active layer were shielded by the GO, the carboxyls beneath the GO nanosheets could still provide a fixed charge and contribute to the higher volume charge density formation.

\subsection{Anti-fouling property enhancement}

Fouling tests were conducted on the pDA/TMC, pDA/TMC/GO10 and $\mathrm{pDA} / \mathrm{TMC} / \mathrm{C} 10$ membranes with synthetic wastewater containing $\mathrm{Ca}^{2+}$ /organic compound foulants, as organic foulants and calcium ions often coexist in wastewater secondary effluent or some natural water (Lu et al., 2013). Calcium and other multivalent cations have been reported to accelerate the fouling process by 'bridge' between the carboxyl groups of the polyamide membrane surface and alginate (Mo et al. 2011, 2012). The pDA/TMC/GO10 membrane exhibited a slower normalized flux decline than the pDA/TMC and pDA/TMC/C10 membranes (Fig. 6). At the end of the fouling tests, the normalized flux of the pDA/TMC/GO10 membrane decreased by $19.0 \%$, which is considerably less than those from the pDA/TMC (36.0\%) and pDA/TMC/C10 (48.8\%) membranes. These results suggest that the membranes became more resistant to fouling after the covalent-binding of the GO to the membrane surface. The fouling reversibility of the GO functionalized membrane (18.0\%) was also higher than those of the pDA/TMC and pDA/
TMC/C10 membranes ( $<1 \%$ ). Hence, the anti-fouling property of the membranes were clearly enhanced after GO shielding.

Membrane characteristics that can influence the anti-fouling properties typically include surface roughness, wettability, surface charge and surface functional groups (Mo et al., 2012; Hobbs et al., 2006; Ni et al., 2014; Wu et al., 2014; Vrijenhoek et al., 2001). The surface roughness and water contact angles of the pDA/TMC membrane were already relatively low. Therefore, the roughness of the membrane surface does not exhibit significant changes after GO functionalization compared with the pDA/TMC membrane (P-Value $>0.05$, Fig. S13), and the water contact angles change of GO modified membranes were also insignificant (PValue $=0.197>0.05$ ) (Fig. S14). These results indicate that compared with the pDA/TMC membrane, the enhanced antifouling property of the GO functionalized membrane in this study do not originate from changes in the surface roughness and wettability. For the surface charge, it has been suggested that a more negatively charged membrane zeta potential may lead to greater repulsion to negatively charged foulants (Mo et al., 2012). The sodium alginate was negatively charged (Contreras et al., 2011). However, because the GO functionalization membrane's zeta potential was more positively charged (Fig. 4e), the repulsion became lower. It meant that the surface charge change could not be the reason for the enhancement of the membrane anti-fouling properties. Moreover, the membrane zeta potential difference will be compressed to minimum by $\mathrm{Ca}^{2+}$ or other multivalent cations and can be ignored (Mo et al., 2012). For the surface functional groups, previous findings indicate that membranes with more $-\mathrm{COOH}$ surface groups attract more alginate in the initial conditioning layer than other groups (Wu et al., 2014). After carboxyls were shielded by the GO nanosheets (Fig. 4), the calcium bridging between the carboxyl groups of alginate molecules and those of the membrane surface were weakened. Therefore, compared with the pDA/TMC membrane, fewer carboxyls appear on the membrane surface after shielded by GO was the most likely reason for the enhancement of anti-fouling properties.

\subsection{Reasons for the increase in water permeability}

As in Figs. 5a and 7 showed that the water permeability of the GO functionalized membrane (pDA/TMC/GO10) $(103.69 \pm 12.04 \mathrm{~L} /$ $\left.\left(\mathrm{m}^{2} \cdot \mathrm{h} \cdot \mathrm{MPa}\right)\right)$ increased by $84.2 \%$ compared with that of the pDA/ TMC membrane $\left(56.28 \pm 18.18 \mathrm{~L} /\left(\mathrm{m}^{2} \cdot \mathrm{h} \cdot \mathrm{MPa}\right)\right)$. However, this increase in water permeability cannot be attributed to the GO functionalization because the water permeability of the control membrane (pDA/TMC/C10) also increased (Figs. 5a and 7). The reason for increase in water permeability was membrane active layer becoming harder and thinner after the EDC/NHS catalyzed.

$\mathrm{EDC} / \mathrm{NHS}$ is widely used to catalyze amide-crosslinks between carboxyl groups and amino groups (Wissink et al., 2001). Fig. 4b, c and Table S1 illustrated that the $-\underline{\mathrm{C}}-\mathrm{N}-$ and $-\underline{\mathrm{C}}(=\mathrm{O})-\mathrm{NH}-$ peaks of pDA/TMC/C10 membrane increased after catalyzed by the EDC/ NHS compared with the pDA/TMC membrane. The $-\mathrm{COOH}$ decrease agreed with $-\underline{\mathrm{C}}(=\mathrm{O})-\mathrm{NH}-$ increase well $(\sim 0.9 \%$ for both, Table S1). These results indicate that a small proportion of the $-\mathrm{COOH}$ on the $\mathrm{pDA} / \mathrm{TMC}$ active layer formed to amide bonds. The more amide-crosslinks formed made membrane active layer becoming harder, which can be supported by the results from the membrane compaction tests (Fig. 7 and S11). The active layer surface pore collapse is one of the key mechanisms for membrane compaction (Hussain et al., 2013). As the compaction tests (Fig. 7 and S11) showed, membranes water permeability all sharply decreased at the beginning of the test, indicating that the membranes were compacted rapidly. However, the stable fluxes of the $\mathrm{pDA} / \mathrm{TMC} / \mathrm{C} 10$ and $\mathrm{pDA} / \mathrm{TMC} / \mathrm{GO} 10$ were notably higher than that 

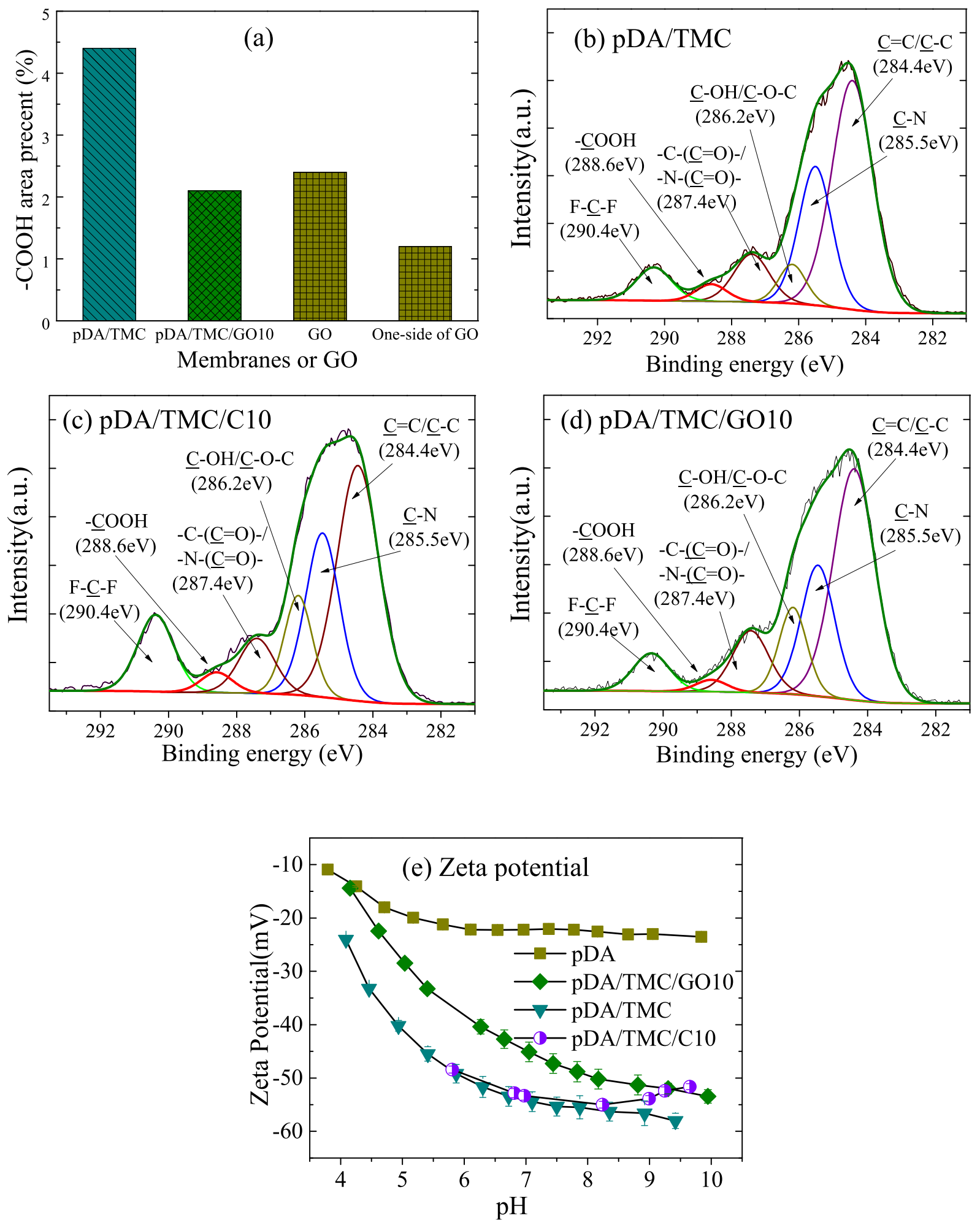

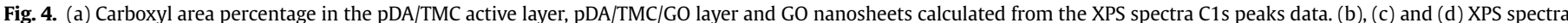

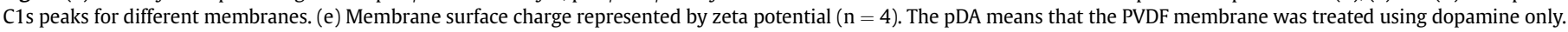

of pDA/TMC membrane. These results revealed the formation of a harder pDA/TMC active layer after catalyzed by the EDC/NHS.

The $-\mathrm{C}-\mathrm{F}$ peak increased after the EDC/NHS treatment based on the XPS C1s data (Fig. 4b, c and S10), which could indicate the pDA/ TMC layer became thinner. The $-\mathrm{C}-\mathrm{F}$ peak could only originate from PVDF supporting layer. For the same X-ray excitation energy, the ability of the excited state electron in the PVDF layer to escape away from the membrane surface is related to the thickness of the pDA/TMC layer above the PVDF layer (Karan et al., 2015). The $-\underline{C}-\mathrm{F}$ peak increase meant more excited state electrons belonging to the PVDF layer escaped from the membrane surface. That revealed a thinner pDA/TMC layer formed. 

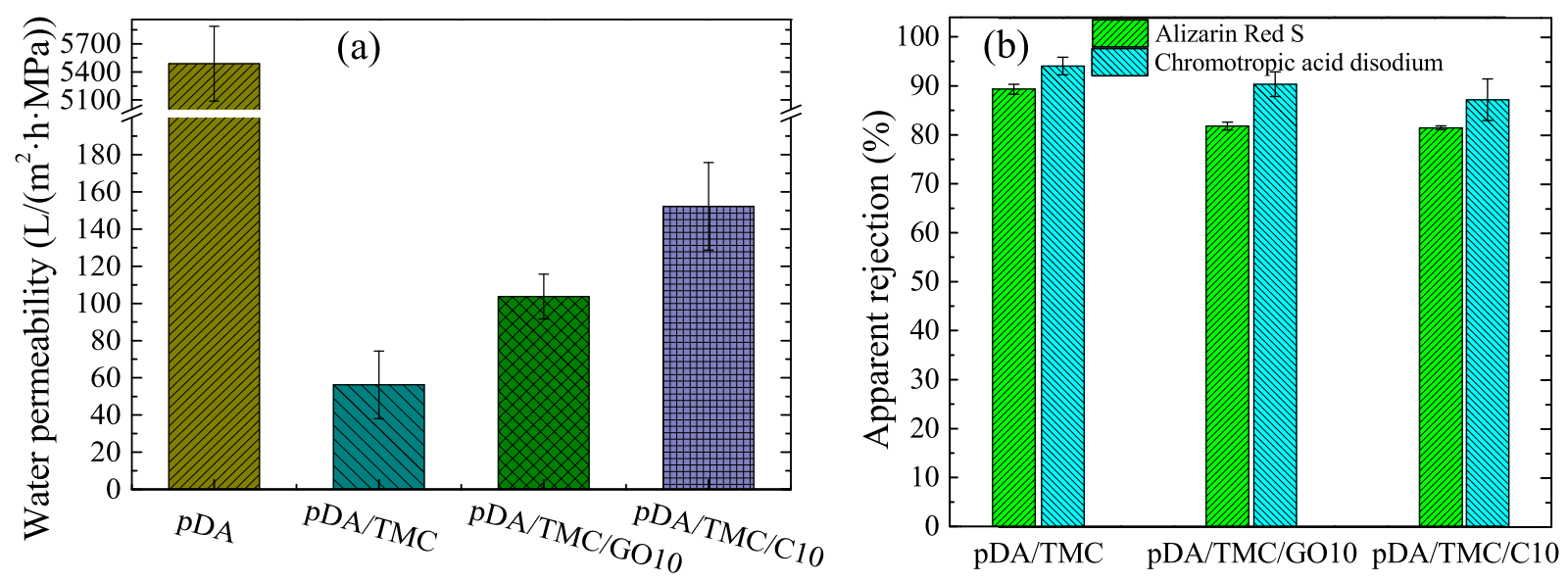

Fig. 5. (a) Water permeability of different membranes $(n=3)$ and (b) apparent rejection data for different membranes to different organic compounds.

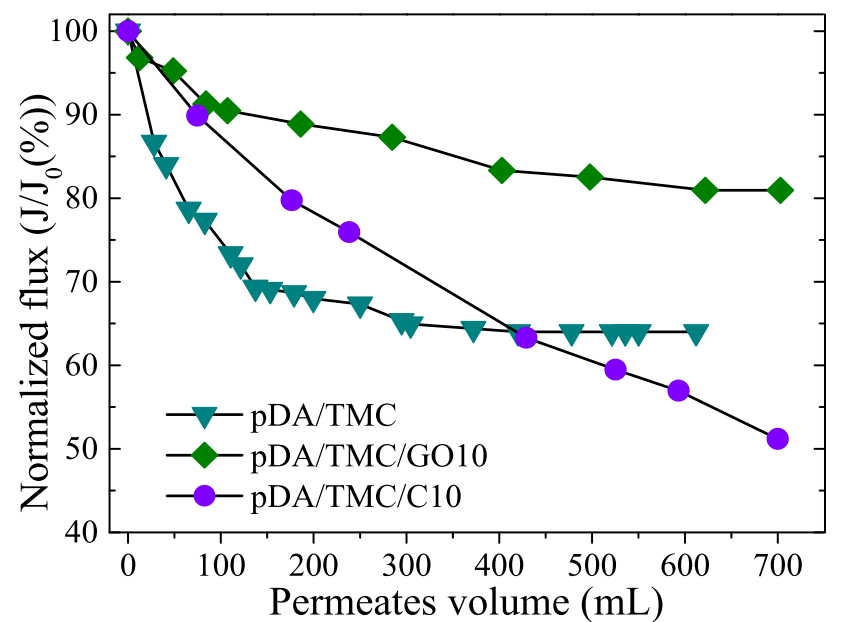

Fig. 6. Anti-fouling property to $\mathrm{Ca}^{2+}$ and alginate compound fouling. J0 is the stable flux prior to the fouling stage, and $\mathrm{J}$ is the real-time flux during the tests. The antifouling property tests were performed with an electrolyte solution containing $1 \mathrm{mM}$ $\mathrm{CaCl}_{2}, 16 \mathrm{mM} \mathrm{NaCl}, 1 \mathrm{mM} \mathrm{NaHCO}$ and $20 \mathrm{mg} / \mathrm{L}$ sodium alginate. The cross membrane pressure was $0.45 \mathrm{MPa}$ with a cross-flow rate of $0.11 \mathrm{~m} / \mathrm{s}$.

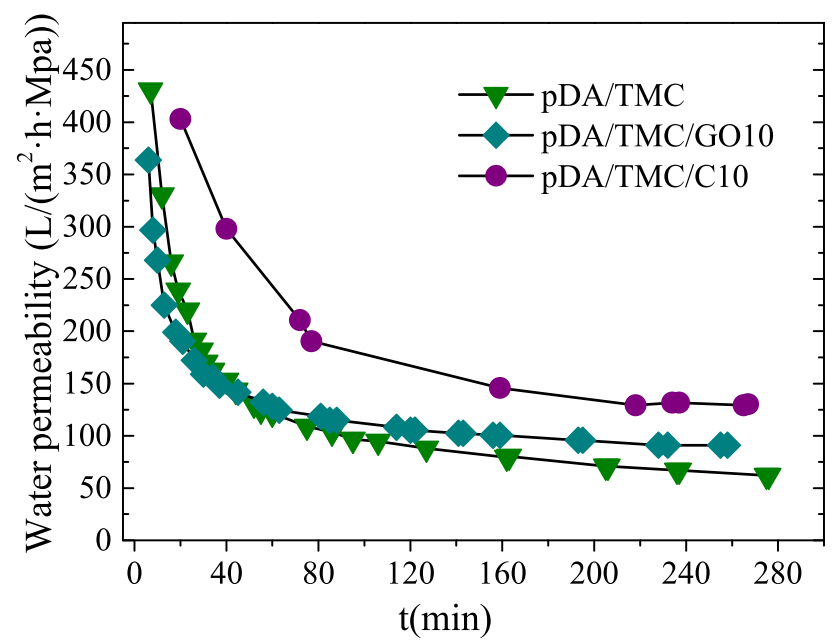

Fig. 7. Water permeability change for different membranes in the early compaction stage. All of the membrane compaction tests were performed under $0.45 \mathrm{MPa}$ with a cross-flow rate of $0.132 \mathrm{~m} / \mathrm{s}$.
The cross-sectional TEM views of different membranes (Fig. $2 \mathrm{~g}-\mathrm{h}$ ) gave direct evidence that pDA/TMC layer became thinner. The thickness of pDA/TMC active layer decreased from 8.9-19.6 $\mathrm{nm}$ to $7.2-12.7 \mathrm{~nm}$ after EDC/NHS treatment.

As the membrane active layer becomes harder and thinner, the surface density of nanopores increased and the collapse of nanopores was eased, thus the water flow path became shorter. All of these resulted in an increase in membrane water permeability.

\subsection{Importance of active layer carboxyls shielding and simultaneous promotion of flux along with anti-fouling property}

The special features of the GO (Hegab and Zou, 2015; Nair et al., 2012; Lee et al., 2008; Hu et al., 2010; Tu et al., 2013; Lui et al., 2009; Novoselov et al., 2004; Dreyer et al., 2010; Hummers and Offeman, 1958; Drioli et al., 2015) make it to an excellent surface modification material. The GO surface modification can effectively exert the good separation properties of organic polymer active layer, and the chlorine resistance and anti-fouling properties of a GO layer simultaneously. A profound understanding of the relationship between the membrane surface functional groups change and the anti-fouling properties change after the GO surface modification is important to the development of GO surface modification. In this work, we proved that by shielding, but not removing the carboxyl groups on the membrane active layer using GO led to improvements in the membrane anti-fouling property. These results are important to multivalent cations/organic foulants compound fouling control, during the membrane purification of natural water and wastewater secondary effluent. Future studies could focus on preparation and selection of GO with different properties, and use them for different modifications purposes.

People are continuously working on pursuing membranes with higher flux. However, higher water permeability means a higher concentration polarization effect for the same operating conditions (Cohen-Tanugi et al., 2014), implying more serious membrane fouling (Bacchin et al., 2006). Our fouling tests (Fig. 6) revealed that flux decline rate of the flux-improved membrane (pDA/TMC/C10) was faster than that for the low flux membrane (pDA/TMC) because the concentration polarization effect increased. This effect significantly offset the amount of flux increment. However, when we promote the flux along with anti-fouling property (pDA/TMC) G010), the flux after fouling is clearly increased, although the concentration polarization effect also increased compared with the low-flux membrane (pDA/TMC). These results reveal the importance of increasing membrane flux and anti-fouling properties 
simultaneously, from a practical application perspective.

\section{Conclusions}

In this work, GO nanosheets were covalently-bound to the surface of a polydopamine/TMC membrane using an environmentally friendly, layer-by-layer self-assembly method, aided by EDC/ NHS-mediated interfacial polymerization. We found that the high increase of membrane anti-fouling properties after GO modification can also result from shielding active layer carboxyl groups by GO nanosheets, apart from previously reported increase in hydrophilicity and reduction in membrane surface roughness. The GO covalently-binding method we used effectually protected the volume charge density of active layer, so the membrane rejection efficiency maintained relatively stable. The EDC/NHS catalysis made the active layer of polydopamine/TMC membrane became thinner and harder by enhancing the amide-crosslinks between carboxylic and amino groups. Membrane flux obviously increased along with GO covalent-binding process.

\section{Funding sources}

Funding sources include the Natural Science Foundation for the Youth (21507150), Distinguished Young Scholars (51225802), the Major Science and Technology Program for Water Pollution Control and Treatment (2014ZX07204-005), the "Hundred Talents Program" of the Chinese Academy of Sciences, Project 135 of Chinese Academy of Sciences (YSW2013B06), the Science and Technology Service Network Initiative of Chinese Academy of Sciences (KFJEW-STS-102) and the Special Fund of the State Key Joint Laboratory of Environment Simulation and Pollution Control (No. 15K10ESPCT).

\section{Acknowledgments}

The authors would like to thank Mr. Wei Wang (MIR\&RAMAN Manager of North China, Brook Technology Co., Ltd (Beijing)) for the infrared spectroscopy technology, Dr. Kang Xiao, Dr. Bo Zhang, Dr. Wen-Li Jiang, Dr. Li-Ming Yang, Dr. Kun Li and Prof. Yuan-Song Wei for their kindly help or advice, Dr. Zhen-Li Sun for providing of Au colloid, and Prof. Menachem Elimelech for the useful discussions.

\section{Appendix A. Supplementary data}

Supplementary data related to this article can be found at http:// dx.doi.org/10.1016/j.watres.2016.06.032.

\section{References}

An, Q., Li, F., Ji, Y., Chen, H., 2011. Influence of polyvinyl alcohol on the surface morphology, separation and anti-fouling performance of the composite polyamide nanofiltration membranes. J. Membr. Sci. 367 (1-2), 158-165.

Bacchin, P., Aimar, P., Field, R.W., 2006. Critical and sustainable fluxes: theory, experiments and applications. J. Membr. Sci. 281 (1-2), 42-69.

Ben-Sasson, M., Lu, X., Bar-Zeev, E., Zodrow, K.R., Nejati, S., Qi, G., Giannelis, E.P., Elimelech, M., 2014. In situ formation of silver nanoparticles on thin-film composite reverse osmosis membranes for biofouling mitigation. Water Res. $62(0), 260-270$.

Berean, K.J., Ou, J.Z., Nour, M., Field, M.R., Alsaif, M.M.Y.A., Wang, Y., Ramanathan, R. Bansal, V., Kentish, S., Doherty, C.M., Hill, A.J., McSweeney, C., Kaner, R.B. Kalantar-zadeh, K., 2015. Enhanced gas permeation through graphene nanocomposites. J. Phys. Chem. C. 119 (24), 13700-13712.

Bowen, W.R., Mukhtar, H., 1996. Characterisation and prediction of separation performance of nanofiltration membranes. J. Membr. Sci. 112 (2), 263-274.

Buksek, H., Luxbacher, T., Petrinic, I., 2010. Zeta potential determination of polymeric materials using two differently designed measuring cells of an electrokinetic analyzer. Acta Chim. Slov. 57 (3), 700-706.

Chae, H.-R., Lee, J., Lee, C.-H., Kim, I.-C., Park, P.-K., 2015. Graphene oxide-embedded thin-film composite reverse osmosis membrane with high flux, anti-biofouling, and chlorine resistance. J. Membr. Sci. 483, 128-135.
Childress, A.E., Elimelech, M., 1996. Effect of solution chemistry on the surface charge of polymeric reverse osmosis and nanofiltration membranes. J. Membr. Sci. 119 (2), 253-268.

Childress, A.E., Elimelech, M., 2000. Relating nanofiltration membrane performance to membrane charge (electrokinetic) characteristics. Environ. Sci. Technol. 34 (17), 3710-3716.

Choi, W., Choi, J., Bang, J., Lee, J.H., 2013. Layer-by-layer assembly of graphene oxide nanosheets on polyamide membranes for durable reverse-osmosis applications. ACS Appl. Mater Interfaces 5 (23), 12510-12519.

Cohen-Tanugi, D., McGovern, R.K., Dave, S.H., Lienhard, J.H., Grossman, J.C., 2014. Quantifying the potential of ultra-permeable membranes for water desalination. Energy \& Environ. Sci. 7 (3), 1134-1141.

Contreras, A.E., Steiner, Z., Miao, J., Kasher, R., Li, Q., 2011. Studying the role of common membrane surface functionalities on adsorption and cleaning of organic foulants using QCM-D. Environ. Sci. Technol. 45 (15), 6309-6315.

Das, A. Chakraborty, B., Sood, A.K., 2008. Raman spectroscopy of graphene on different substrates and influence of defects. Bull. Mater. Sci. 31 (3), 579-584.

Dreyer, D.R., Park, S., Bielawski, C.W., Ruoff, R.S., 2010. The chemistry of graphene oxide. Chem. Soc. Rev. 39 (1), 228-240.

Drioli, E., Ali, A., Macedonio, F., 2015. Membrane distillation: recent developments and perspectives. Desalination 356, 56-84.

Han, Y., Jiang, Y., Gao, C., 2015. High-flux graphene oxide nanofiltration membrane intercalated by carbon nanotubes. ACS Appl. Mater Interfaces 7 (15), 8147-8155.

Hegab, H.M., Zou, L., 2015. Graphene oxide-assisted membranes: fabrication and potential applications in desalination and water purification. J. Membr. Sci. 484, 95-106.

Hegab, H.M., Wimalasiri, Y., Ginic-Markovic, M., Zou, L., 2015. Improving the fouling resistance of brackish water membranes via surface modification with graphene oxide functionalized chitosan. Desalination 365, 99-107.

Hobbs, C., Taylor, J., Hong, S., 2006. Effect of surface roughness on fouling of RO and NF membranes during filtration of a high organic surficial groundwater. J. Water Supply Res. Technol.-AQUA 55 (7-8), 559.

Hong, S.K., Elimelech, M., 1997. Chemical and physical aspects of natural organic matter (NOM) fouling of nanofiltration membranes. J. Membr. Sci. 132 (2), $159-181$.

Hu, M., Mi, B., 2013. Enabling graphene oxide nanosheets as water separation membranes. Environ. Sci. Technol. 47 (8), 3715-3723.

Hu, W.B., Peng, C., Luo, W.J., Lv, M., Li, X.M., Li, D., Huang, Q., Fan, C.H., 2010. Graphene-based antibacterial paper. Acs Nano 4 (7), 4317-4323.

Hu, M., Zheng, S., Mi, B., 2016. Organic fouling of graphene oxide membranes and its implications for membrane fouling control in engineered osmosis. Environ. Sci. Technol. 50 (2), 685-693.

Hummers, W.S., Offeman, R.E., 1958. Preparation of graphitic oxide. J. Am. Chem. Soc. 80 (6), 1339-1339.

Hung, W.-S., Tsou, C.-H., De Guzman, M., An, Q.-F., Liu, Y.-L., Zhang, Y.-M., Hu, C.-C., Lee, K.-R., Lai, J.-Y., 2014. Cross-linking with diamine monomers to prepare composite graphene oxide-framework membranes with varyingd-spacing. Chem. Mater. 26 (9), 2983-2990.

Hussain, Y.A., Al-Saleh, M.H., Ar-Ratrout, S.S., 2013. The effect of active layer nonuniformity on the flux and compaction of TFC membranes. Desalination 328, $17-23$.

Jie, G., Kongyin, Z., Xinxin, Z., Zhijiang, C., Min, C., Tian, C., Junfu, W., 2015. Preparation and characterization of carboxyl multi-walled carbon nanotubes/calcium alginate composite hydrogel nano-filtration membrane. Mater. Lett. 157, $112-115$.

Karan, S., Jiang, Z., Livingston, A.G., 2015. Sub-10 nm polyamide nanofilms with ultrafast solvent transport for molecular separation. Science 348 (6241), 1347-1351.

Lee, C., Wei, X., Kysar, J.W., Hone, J., 2008. Measurement of the elastic properties and intrinsic strength of monolayer graphene. Science 321 (5887), 385-388.

Li, X., Cai, T., Chen, C., Chung, T.S., 2016. Negatively charged hyperbranched polyglycerol grafted membranes for osmotic power generation from municipal wastewater. Water Res. 89, 50-58.

Liu, Y., Ai, K., Lu, L., 2014. Polydopamine and its derivative materials: synthesis and promising applications in energy, environmental, and biomedical fields. Chem. Rev. 114 (9), 5057-5115.

Liu, M., Chen, Q., Wang, L., Yu, S., Gao, C., 2015. Improving fouling resistance and chlorine stability of aromatic polyamide thin-film composite RO membrane by surface grafting of polyvinyl alcohol (PVA). Desalination 367, 11-20.

Lu, X., Romero-Vargas Castrillon, S., Shaffer, D.L., Ma, J., Elimelech, M., 2013. In situ surface chemical modification of thin-film composite forward osmosis membranes for enhanced organic fouling resistance. Environ. Sci. Technol. 47 (21), 12219-12228.

Lui, C.H., Liu, L., Mak, K.F., Flynn, G.W., Heinz, T.F., 2009. Ultraflat graphene. Nature 462 (7271), 339-341.

Marcano, D.C., Kosynkin, D.V., Berlin, J.M., Sinitskii, A., Sun, Z.Z., Slesarev, A., Alemany, L.B., Lu, W., Tour, J.M., 2010. Improved synthesis of graphene oxide. Acs Nano 4 (8), 4806-4814.

Mo, Y., Xiao, K., Shen, Y., Huang, X., 2011. A new perspective on the effect of complexation between calcium and alginate on fouling during nanofiltration. Sep. Purif. Technol. 82, 121-127.

Mo, Y., Tiraferri, A., Yip, N.Y., Adout, A., Huang, X., Elimelech, M., 2012. Improved antifouling properties of polyamide nanofiltration membranes by reducing the density of surface carboxyl groups. Environ. Sci. Technol. 46 (24), 13253-13261. 
Nair, R.R., Wu, H.A., Jayaram, P.N., Grigorieva, I.V., Geim, A.K., 2012. Unimpeded permeation of water through helium-leak-tight graphene-based membranes. Science 335 (6067), 442-444.

Ni, L., Meng, J.Q., Li, X.G., Zhang, Y.F., 2014. Surface coating on the polyamide TFC RO membrane for chlorine resistance and antifouling performance improvement J. Membr. Sci. 451, 205-215.

Novoselov, K.S., Geim, A.K., Morozov, S.V., Jiang, D., Zhang, Y., Dubonos, S.V., Grigorieva, I.V., Firsov, A.A., 2004. Electric field effect in atomically thin carbon films. Science 306 (5696), 666-669.

Peng, J., Su, Y., Chen, W., Zhao, X., Jiang, Z., Dong, Y., Zhang, Y., Liu, J., Xingzhong, C., 2013. Polyamide nanofiltration membrane with high separation performance prepared by EDC/NHS mediated interfacial polymerization. J. Membr. Sci. 427, 92-100.

Perreault, F., Tousley, M.E., Elimelech, M., 2014. Thin-film composite polyamide membranes functionalized with biocidal graphene oxide nanosheets. Environ. Sci. Technol. Lett. 1 (1), 71-76.

Perreault, F., Fonseca de Faria, A., Elimelech, M., 2015. Environmental applications of graphene-based nanomaterials. Chem. Soc. Rev. 44 (16), 5861-5896.

Petersen, R.J., 1993. Composite reverse osmosis and nanofiltration membranes. J. Membr. Sci. 83 (1), 81-150.

Riddick, T.M., 1968. Control of colloid stability through zeta potential. Blood 10, 1.

Sanyal, O., Lee, I., 2014. Recent progress in the applications of layer-by-layer assembly to the preparation of nanostructured ion-rejecting water purification membranes. J. Nanosci. Nanotechnol. 14 (3), 2178-2189.

Staros, J.V., Wright, R.W., Swingle, D.M., 1986. Enhancement by N-hydroxysulfosuccinimide of water-soluble carbodiimide-mediated coupling reactions. Anal. Biochem. 156 (1), 220-222.

Sun, Z., Du, J., Yan, L., Jing, C., 2014. Rapid detection of 2,2',4,4'-tetrabromodiphenyl ether (BDE-47) using a portable Au-colloid SERS sensor. J. Raman Spectrosc. 45 (9), 745-749.

Szymczyk, A., Fievet, P., 2005. Investigating transport properties of nanofiltration membranes by means of a steric, electric and dielectric exclusion model. J. Membr. Sci. 252 (1-2), 77-88.

Tiraferri, A., Vecitis, C.D., Elimelech, M., 2011. Covalent binding of single-walled carbon nanotubes to polyamide membranes for antimicrobial surface properties. Acs Appl. Mater. Interfaces 3 (8), 2869-2877.

Tu, Y., Lv, M., Xiu, P., Huynh, T., Zhang, M., Castelli, M., Liu, Z., Huang, O., Fan, C.,
Fang, H., Zhou, R., 2013. Destructive extraction of phospholipids from Escherichia coli membranes by graphene nanosheets. Nat. Nanotechnol. 8 (8), 594-601.

Van Wagner, E.M., Sagle, A.C., Sharma, M.M., La, Y.-H., Freeman, B.D., 2011. Surface modification of commercial polyamide desalination membranes using poly(ethylene glycol) diglycidyl ether to enhance membrane fouling resistance. Membr. Sci. 367 (1-2), 273-287.

Vezzani, D., Bandini, S., 2002. Donnan equilibrium and dielectric exclusion for characterization of nanofiltration membranes. Desalination 149 (1-3), $477-483$.

Vrijenhoek, E.M., Hong, S., Elimelech, M., 2001. Influence of membrane surface properties on initial rate of colloidal fouling of reverse osmosis and nanofiltration membranes. J. Membr. Sci. 188 (1), 115-128.

Wissink, M., Beernink, R., Pieper, J., Poot, A., Engbers, G., Beugeling, T., Van Aken, W. Feijen, J., 2001. Immobilization of heparin to EDC/NHS-crosslinked collagen. Characterization and in vitro evaluation. Biomaterials 22 (2), 151-163.

Wu, J.J., Contreras, A.E., Li, Q.L., 2014. Studying the impact of RO membrane surface functional groups on alginate fouling in seawater desalination. J. Membr. Sci. 458, 120-127.

Xie, M., Lee, J., Nghiem, L.D., Elimelech, M., 2015. Role of pressure in organic fouling in forward osmosis and reverse osmosis. J. Membr. Sci. 493, 748-754.

Ye, G., Lee, J., Perreault, F., Elimelech, M., 2015. Controlled architecture of dualfunctional block copolymer brushes on thin-film composite membranes for integrated "Defending" and "Attacking" strategies against biofouling. ACS Appl. Mater Interfaces 7 (41), 23069-23079.

Yu, B., Shu, X., Cong, H., Chen, X., Liu, H., Yuan, H., Chi, M., 2016. Self-assembled covalent capillary coating of diazoresin/carboxyl fullerene for analysis of proteins by capillary electrophoresis and a comparison with diazoresin/graphene oxide coating. J. Chromatogr. A 1437, 226-233.

Zhao, D., Yu, S., 2014. A review of recent advance in fouling mitigation of NF/RO membranes in water treatment: pretreatment, membrane modification, and chemical cleaning. Desalin. Water Treat. 55 (4), 870-891.

Zhao, J.J., Su, Y.L., He, X., Zhao, X.T., Li, Y.F., Zhang, R.N., Jiang, Z.Y., 2014. Dopamine composite nanofiltration membranes prepared by self-polymerization and interfacial polymerization. J. Membr. Sci. 465, 41-48.

Zou, X., Zhang, L., Wang, Z., Luo, Y., 2016. Mechanisms of the antimicrobial activities of graphene materials. J. Am. Chem. Soc. 138 (7), 2064-2077. 